\title{
Regulation of leukotriene and 5oxoETE synthesis and the effect of 5-lipoxygenase inhibitors: a mathematical modeling approach
}

\author{
Tatiana A Karelina ${ }^{1 *}$, Kirill V Zhudenkov , Oleg O Demin', Dmitry V Svetlichny ${ }^{1}$, Balaji Agoram², \\ David Fairman ${ }^{3,4}$ and Oleg $\vee$ Demin $^{1}$
}

\begin{abstract}
Background: 5-lipoxygenase (5-LO) is a key enzyme in the synthesis of leukotrienes and 5-Oxo-6E,8Z,11Z,14Zeicosatetraenoic acid (oxoETE). These inflammatory signaling molecules play a role in the pathology of asthma and so 5-LO inhibition is a promising target for asthma therapy. The 5-LO redox inhibitor zileuton (Zyflo IR/CR ${ }^{\circledR}$ ) is currently marketed for the treatment of asthma in adults and children, but widespread use of zileuton is limited by its efficacy/safety profile, potentially related to its redox characteristics. Thus, a quantitative, mechanistic description of its functioning may be useful for development of improved anti-inflammatory targeting this mechanism.
\end{abstract}

Results: A mathematical model describing the operation of 5-LO, phospholipase A2, glutathione peroxidase and 5-hydroxyeicosanoid dehydrogenase was developed. The catalytic cycles of the enzymes were reconstructed and kinetic parameters estimated on the basis of available experimental data. The final model describes each stage of cys-leukotriene biosynthesis and the reactions involved in oxoETE production. Regulation of these processes by substrates (phospholipid concentration) and intracellular redox state (concentrations of reduced glutathione, glutathione (GSH), and lipid peroxide) were taken into account. The model enabled us to reveal differences between redox and non-redox 5-LO inhibitors under conditions of oxidative stress. Despite both redox and non-redox inhibitors suppressing leukotriene A4 (LTA4) synthesis, redox inhibitors are predicted to increase oxoETE production, thus compromising efficacy. This phenomena can be explained in terms of the pseudo-peroxidase activity of 5-LO and the ability of lipid peroxides to transform 5-LO into its active form even in the presence of redox inhibitors.

Conclusions: The mathematical model developed described quantitatively different mechanisms of 5-LO inhibition and simulations revealed differences between the potential therapeutic outcomes for these mechanisms.

\section{Background}

Leukotrienes are key inflammatory mediators associated with pathological states of inflammation in diseases such as asthma and allergic rhinitis and play a pivotal role in normal host defense [1]. They have been shown to promote leukocyte chemotaxis and activation, vascular tone and permeability, smooth muscle contractility and immune function. 5-lipoxygenase (5-LO) is the key enzyme

\footnotetext{
* Correspondence: karelina@insysbio.ru

'Institute for Systems biology SPb, Moscow, Russia

Full list of author information is available at the end of the article
}

of leukotriene biosynthesis and so is a promising target for drug development $[2,3]$.

5-LO is expressed predominantly in leukocytes and is responsible for the synthesis of both leukotriene A4 (LTA4) and 5(S)-hydroperoxy-6,8,1l,14-(E,Z,Z,Z)-eicosatetraenoic acid (HP) [4-6]. The reaction scheme is given in Figure 1. There are two steps in this reaction: oxygenation of arachidonic acid (AA) using $\mathrm{O}_{2}$ to produce $\mathrm{HP}$ and the dehydration of the hydroperoxide intermediate, to produce the epoxide, leukotriene A4 (LTA4). HP can be further converted either to 5-hydroxyeicosatetraenoic acid (HT) by glutathione peroxidase (GPx) $[7,8]$. HT, in turn, 


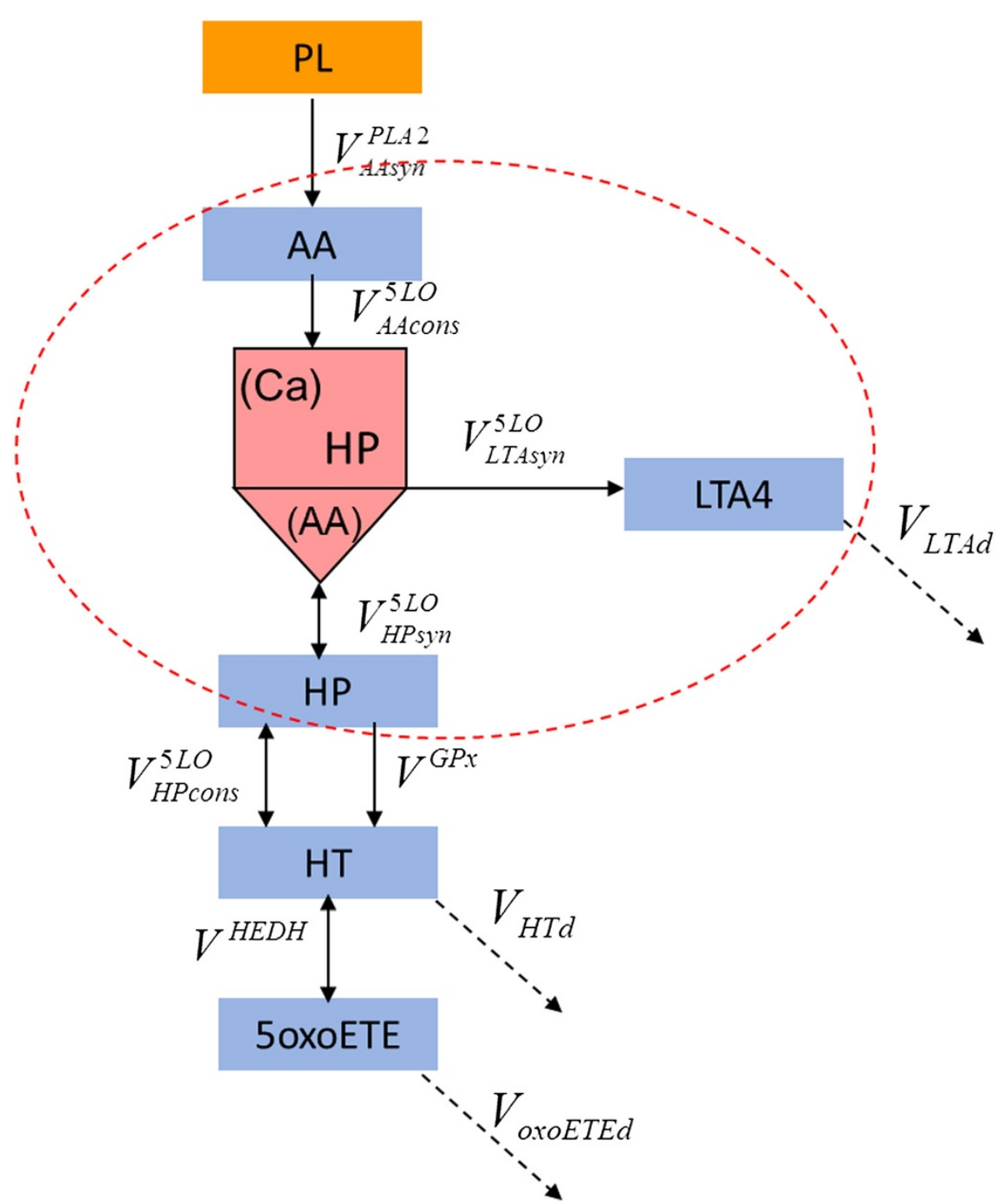

Figure 1 Schematic representation of Leukotriene and oxoETE synthesis model ("LOS model"). The reactions occurring with 5-LO are in the dashed red circle. Blue rectangular represents metabolites which are variables of "LOS model". Pink colour indicates complex of 5-LO and HP. Dashed arrows stand for degradation processes.

can be converted to 5-Oxo-6E,8Z,11Z,14Z-eicosatetraenoic acid (oxoETE) by 5-hydroxyeicosanoid dehydrogenase $(\mathrm{HEDH})[9]$. oxoETE is produced by various cells including neutrophils, eosinophils, and monocytes [10] and acts as a potent chemo-attractant for these cell types. For example, 5oxoETE stimulates eosinophil migration and tissue infiltration 30 fold more potently than leukotriene B4 (LTB4) [11], and also increases intracellular calcium $\left(\mathrm{Ca}^{2+}\right)$ concentration and actin polymerization in eosinophils [10].

5 - $\mathrm{LO}$ activity is controlled by the intracellular $\mathrm{Ca}^{2+}$ concentration and the cellular redox state [4-6,12,13]. The redox state modulates activity via the iron (Fe) atom located in the catalytic site of the enzyme. HP and other lipid peroxides are able to oxidize the Fe atom from $\mathrm{Fe}^{2+}$ (ferrous state) to the active $\mathrm{Fe}^{3+}$ (ferric state). Consistent with this the addition of glutathione peroxidase (GPx) inhibits formation of products of 5-LO catalyzed reactions in vitro [13]. Similar to other lipoxygenases, 5-LO also demonstrates redox state dependent hydroperoxidase activity [14]: ferrous 5-LO reacts with lipid-hydroperoxide to form ferric 5-LO, an oxygen-centered lipid radical and hydroxide ion. Additionally, 5-LO activity, in the presence of $\mathrm{Ca}^{2+}$, is increased by structural stabilization via ATP (adenosine-5'-triphosphate) without ATP hydrolysis, microsomal membranes or phosphatidylcholine vesicles (PtdCho) [13]. Mitogen activated protein MAP kinase cascade activity, nuclear import and export of 5-LO, interaction with coactosin-like protein (CLP), 5-LO activating protein (FLAP) and phosphorylation of the 
enzyme by protein kinase A, resulting in suppression of 5-LO activity, also have been reported to modulate it's activity [12].

Most of the known inhibitors of the 5-LO act on the basis of a redox-mechanism or by chelation of the $\mathrm{Fe}$ atom of 5-LO [3]. Redox inhibitors reduce the Fe atom from active ferric state to the inactive ferrous state. The complexity of 5-LO regulation and the multiple reaction paths suggests that redox and non-redox inhibitors may have qualitatively and quantitatively different effects on the products of 5-LO catalyzed reactions.

Zileuton (Z, N-(l-benzo(b)thien-2-ylethyl)-N-hydroxyurea or Zyflo IR/CR ${ }^{\circledR}$ ), is a redox inhibitor of 5-LO currently approved for the daily treatment of asthma in adults and children [15]. Zileuton has a sub-optimal pharmacokinetic and pharmacodynamic profile resulting in a high total daily dose $(2400 \mathrm{mg})$ and frequent dosing (4 times a day [q.i.d] for Zyflo $\mathrm{IR}^{\circledR}$ and twice a day [b.i.d] for Zyflo $\mathrm{CR}^{\circledR}$ ) [16], plus a potential for hepatotoxicity [17]. Therefore, the development of a medicine with more convenient dosing regimen may maximise the benefits of inhibiting the leukotriene pathway and provide efficacy superior to that obtained with zileuton. PF-4191834, 4-(3-(4-(1-methyl-1H-pyrazol-5-yl)phenylthio)phenyl)tetrahydro-2H-pyran-4-carboxamide (PF), is a novel non-iron chelating, non-redox, 5-LO inhibitor under investigation for the treatment of various inflammatory conditions [18]. The presence of significant data in the public domain for zileuton and PF suggested that development of a mathematical model would allow insight into the potential differential effects of non-redox and redox inhibitors. To characterize these potential effects it was necessary to attempt to capture the key properties of the entire pathway and its interactions in inflammatory states Therefore, a detailed mathematical model, describing the processes of 5-LO mediated catalysis regulation (self-inactivation, effect of redox state of the medium) was developed.

Several mathematical models of 5-LO have already been reported, for example a model describing the inhibition of lipoxygenase activity by one of the substrates of the enzyme, AA, by Aharony et al. [19]. In this model, 5LO is able to bind 2 molecules of AA simultaneously (one molecule in the catalytic site and other molecule in the additional regulatory site) which renders the enzyme catalytically inactive. However, LTA synthesis and the pseudo-peroxidase reaction have not been taken into account in this model. An alternative model of reticulocyte lipoxygenase was developed with 9,12(Z,Z)-octadecadienoic acid (linoleic acid) as substrate [20]. This model did take into account both the activation of the enzyme by product, hydroperoxy derivative of fatty acid, and inhibition of 5-LO with substrate, polienoic fatty acid. However, the substrate inhibition was described in terms of a competitive mechanism, binding to inactive form of 5-LO thus preventing its activation, and the LTA4 synthase activity of the enzyme was not captured. Additionally, none of the models describe the reversible inactivation of 5-LO with HT [21] and irreversible inactivation with LTA4 [22].

The rate laws for lipoxygenase and LTA4 synthase reactions were derived by Yang et al. [23], where the influence of various inhibitors of 5-LO and cyclooxygenase on AA metabolism were determined. These rate equations take into account the inhibition of 5 - $\mathrm{LO}$ activity by LTA4, HP and HT. However, the inhibition with LTA4 was also described as reversible and the substrate inhibition, product activation and pseudo-peroxidase activity of 5-LO were not taken into account.

Therefore, an opportunity exists to develop a more detailed model of 5-LO activity which describes all the activities of the enzyme and their regulation by substrates and products. The main purpose of this paper is to summarize the development of a detailed mathematical model of 5-LO operation, its application to describe the production of LTA4 and oxoETE, and to study the differences between redox and non-redox inhibitors. The "LOS (Leukotriene-OxoeETE-Synthesis) model" (Figure 1) includes four enzymes: 5-LO, cytosolic phospholipase A2 (cPLA2), GPx and HEDH and describes the major interactions between the components of the system (for example, the influence of glutathione concentration on 5-LO activity). We used the "LOS model" to predict the dose-responses of various inflammatory mediators to redox and non-redox inhibitors and provide a mechanistic explanation for the differences between them.

\section{Methods}

\section{Model construction}

The "LOS model" describing LTA4 and oxoETE production in leukocytes includes reactions catalyzed by 5-LO, cPLA2, GPx, HEDH and degradation of HT, oxoETE and LTA4. Since all leukotrienes are synthesized from LTA4, analysis of 5-LO catalyzed LTA4 production was deemed sufficient to evaluate the impact of 5-LO on leukotriene production. Catalytic cycles for each of the enzymes were constructed and rate equations describing the dependence of reaction rate on concentrations of substrates, products and effectors were derived utilizing literature data.

\section{The kinetic model of 5-LO Known experimental data and hypotheses used for the model development}

In this study, we used the following available experimental data and facts on structural and functional properties of 5-LO:

1. AA inhibits 5-LO activity in lipoxygenase reaction at high concentrations (substrate inhibition) [24]. 
2. LTA4 can be synthesized from exogenous HP [25].

3. 5-LO is self-inactivating [25]. Glutathione peroxidase and glutathione protect the enzyme from inactivation and lipid peroxides eliminate the protective effect of glutathione.

4. HT is a reversible inhibitor of 5-LO [21].

5. LTA4 can inactivate 5-LO irreversibly [22].

6. The Fe atom in the catalytic site exists in two possible states $\mathrm{Fe}^{2+}$ and $\mathrm{Fe}^{3+}$. $\mathrm{Fe}^{3+}$ is the catalytically active state. Transition between $\mathrm{Fe}^{2+}$ and $\mathrm{Fe}^{3+}$ states proceeds via oxidation by lipid peroxides, including HP [12]. Reduction from $\mathrm{Fe}^{3+}$ to $\mathrm{Fe}^{2+}$ state can be mediated by redox inhibitors (zileuton).

7. Oxidation of the enzyme ( $\mathrm{Fe}^{2+} \rightarrow \mathrm{Fe}^{3+}$ transition) is influenced by $\mathrm{Ca}^{2+}$ ions [25].

8. Endogenously generated 5-HP is the preferential substrate for the 5-LO mediated LTA-synthase reaction [26].

9. ATP and membrane binding are necessary for 5-LO activation [13].

During model building and simplification, the following assumptions were made:

a. There are two sites for AA binding: the catalytic site and regulatory site. HT is a competitive inhibitor at the catalytic site.

b. Binding of AA to the regulatory site results in formation of dead-end complexes with 5-LO.

c. Binding of AA and HT to the catalytic site are fast reactions in comparison to product formation.

d. Oxidation and reduction of 5-LO (transition between $\mathrm{Fe}^{2+}$ and $\mathrm{Fe}^{3+}$ ) can result from (i) interaction with lipid peroxides or (ii) "spontaneously" (by means of interaction with oxidative or reducing factors: $\mathrm{O}_{2}$, $\mathrm{H}_{2} \mathrm{O}_{2}$, thiol groups etc.).

e. $\mathrm{Ca}^{2+}$ is able to bind to the catalytic site, but it influences only oxidation and reduction reactions, thus kinetic parameters of other reactions of catalytic cycle remain unchanged.

f. Binding of $\mathrm{Ca}^{2+}$ and redox-inhibitors (Z) are fast reactions in comparison to the other reactions of the catalytic cycle.

g. Redox inhibitors are only able to bind to the catalytic site of 5-LO and only when it is not occupied with other factors.

h. The binding of redox inhibitors to the catalytic site blocks the binding of other factors .

i. The regulatory site is able to bind AA only if following conditions are fulfilled: (i) $5-\mathrm{LO}$ is in $\mathrm{Fe}^{3+}$ state and (ii) the enzyme is not bound to a redoxinhibitor.

j. The oxygenase, pseudoperoxydase and LTA4 synthase activities of 5-LO were simplified by not describing electron transfer and oxygen binding. Instead, it was assumed that the oxygen concentration is in saturation, i.e., it is not a parameter of the model.

$k$. To decrease the number of unknown parameters in our model we have assumed that binding of AA to the regulatory site of 5-LO does not influence the binding of AA, HP and HT to the catalytic site (see Additional file 1: Appendix 1).

1. Interaction with MAP kinases, FLAP, CLP and transport of 5-LO to the nucleus have not been taken into account in the model.

\section{Catalytic cycle of 5-LO}

Figure 2 shows schematic representation of the enzyme states considered in the model. In the ferric state 5-LO is able to bind any substrates/products/inhibitors at its catalytic and regulatory sites. This state of 5-LO was represented as a square with a triangle underneath the square. The square designates the catalytic site of the enzyme and the triangle represents the regulatory site. The regulatory site can be found in 2 states: free or AA bound. The catalytic site of $5-\mathrm{LO}$ is able to bind AA, HT, HP, PF (non-redox inhibitor), Z (redox inhibitor). As an example Figure 2(a) shows the HT and AA bound enzyme state. All the above mentioned compounds compete for the substrate binding part of the catalytic site. In addition to the substrate/product (AA, HP) and competitive inhibitors (HT, PF and Z) the catalytic site of 5LO is able to bind $\mathrm{Ca}^{2+}$ (as shown in Figure 2(a)) which does not compete with AA, HT, HP, PF and Z.

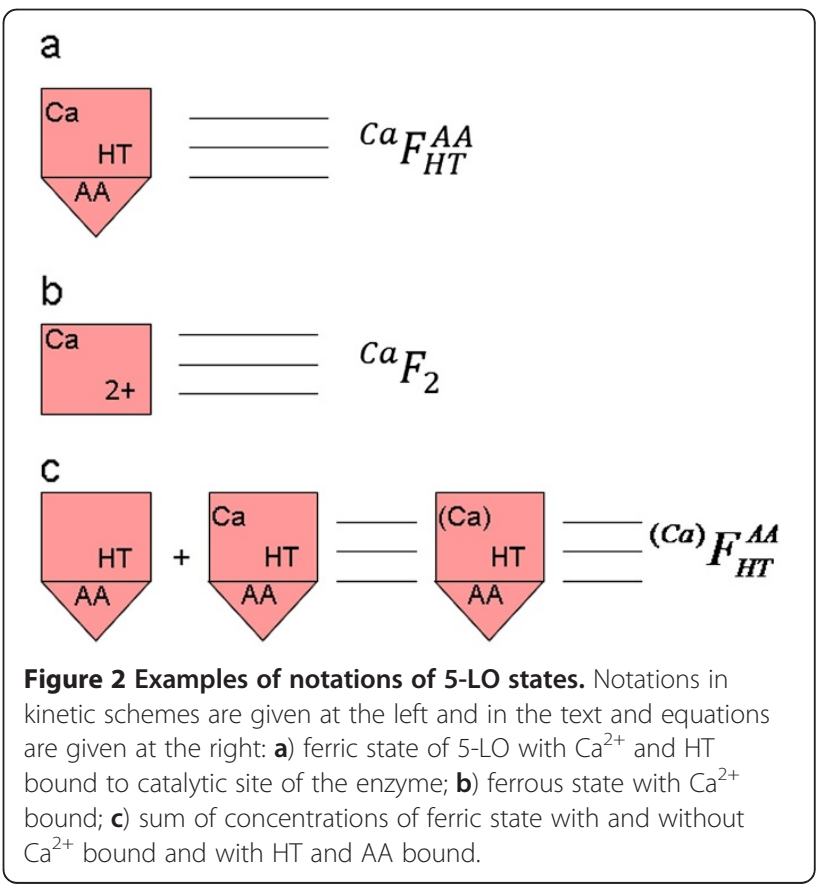


Therefore, the catalytic site of the ferric enzyme can be found in a total of 12 states: free of any substrate/competitor and with AA, HT, HP, PF, Z bound all of which can be found with and without $\mathrm{Ca}^{2+}$ bound. On the basis of this analysis we can conclude that catalytic cycle of 5-LO includes 24 potential states (See Additional file 2: Appendix Figures A1 and A2). The ferrous state $\left(\mathrm{Fe}^{2+}\right)$ of 5-LO is not able to bind any substrate/product/ inhibitor at the catalytic or regulatory site but it is able to bind $\mathrm{Ca}^{2+}$ at the catalytic site (see Figure 2(b)). Thus, total catalytic cycle includes 26 states of 5-LO. Since in derivation of the rate equations describing 5-LO activities we have used new variables representing sums of states of 5-LO, notations for such sums have also been introduced (Figure 2(c)). In the equations and text of this paper we have used the simplified notations of the 5-LO states (see Figure 1).

Transitions between the states are described in accordance to mass action law and can be either reversible (for example, binding AA to catalytic site) or irreversible (for example, the LTA-synthase reaction). Moreover, these processes can be either relatively fast or slow depending on the values of rate constants obtained from experimental data fitting. This grouping of all processes into two sets (fast and slow processes) allowed us to reduce the initial complexity of the catalytic cycle and derive rate equations describing the operation of 5-LO according to the methods described in [27].
Schematic visualization of the total catalytic cycle is not convenient because of the complexity (26 nodes/ states and tenths of transitions between them). To reconstruct a reduced total catalytic cycle and derive rate equations we employed a step-by-step strategy described in Additional file 1: Appendix 1. As a result we have developed a reduced catalytic cycle (Figure 3) describing oxygenation, dehydration and pseudoperoxidase activities of 5-LO. On the basis of this reduced catalytic cycle, we have derived rate equations (1-7) describing 5-LO mediated AA consumption $\left(V_{\text {AAcons }}^{5 L O}\right), 5$-HP production in oxygenase reaction $\left(V_{H P c o n s}^{5 L O}\right), \mathrm{HT}$ production and HP consumption in pseudoperoxidase reaction $\left(V_{H P c o n s}^{5 L O}\right)$, all other lipid peroxide (LOOH) consumption in pseudoperoxidase reaction $\left[V_{L O O H c o n s}^{5 L O}\right]$ and LTA4 production $\left(V_{\text {LTAsyn }}^{5 L O}\right)$

$$
\begin{aligned}
& V_{\text {AAcons }}^{5 L O}=k_{l o} \frac{A A}{K_{A A} \Delta_{Z}} \frac{F_{a}}{\Delta_{t o t}} \\
& V_{\text {LTAsyn }}^{5 L O}=k_{\text {LTAsyn }} \Delta_{H P} \frac{F a}{\Delta_{t o t} \delta} \\
& V_{H P s y n}^{5 L O}=k_{3} \frac{F_{a}}{\Delta_{t o t}}\left(\frac{H P}{\Delta_{Z} K_{d 3}} \delta_{H P-\Delta_{H P}}\right) \\
& V_{\text {HPcons }}^{5 L O}=\frac{F_{a}}{\Delta_{t o t}} \rho_{1}\left(H P \Delta_{\text {redox }}-\frac{H T}{K_{o x}}\right)
\end{aligned}
$$

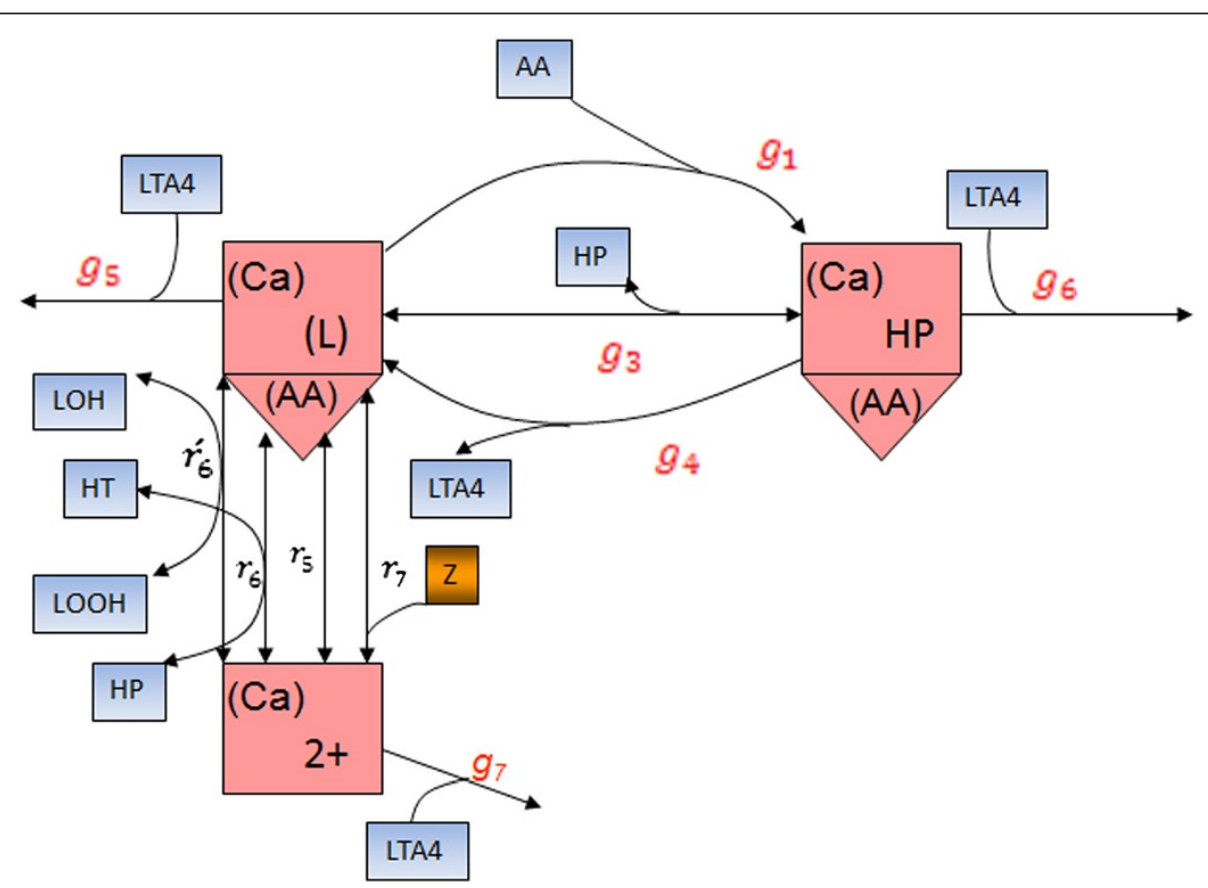

Figure 3 Reduced catalytic cycle of 5-LO. Blue rectangular represents metabolites which are variables of "LOS model". Designation of enzyme states corresponds to Figure 2. Letters with indexes designate effective rate constants (see Additional file 1: Appendix 1). 


$$
\begin{aligned}
& V_{H P s y n}^{5 L O}=V_{H P c o n s}^{5 L O} \\
& V_{L O O H c o n s}^{5 L O}=\frac{F_{a}}{\Delta_{t o t}} \rho_{1}\left(L O O H \Delta_{r e d o x}-\frac{L O H}{K_{o x}}\right) \\
& V_{\text {LOHsyn }}^{5 L O}=V_{\text {LOOHcons' }}^{5 L O}
\end{aligned}
$$

Where

$$
\begin{aligned}
& \Delta_{t o t}=\delta L+\Delta_{H P}+\Delta_{\text {redox }} \\
& \Delta H P=\frac{k_{l o} \frac{A A}{k_{L T A s y n}}+\frac{k_{3}}{k_{d 3}} H P \delta H P}{\Delta_{Z}\left(\frac{{ }^{L} L T A s y n}{\delta H P}+k_{3}\right)} \\
& \delta_{L}=1+\frac{A A}{K_{A A}^{i} \Delta_{Z}}+\delta H P\left(\frac{A A}{K_{A A}}+\frac{H T}{K_{H T}}+\frac{P F}{K_{P F}}\right) \frac{1}{\Delta_{Z}} \\
& \delta H P=1+\frac{A A}{k^{i}{ }_{A A}} \\
& \Delta_{\text {redox }}=\frac{\frac{\rho_{2}}{K_{r}}+\frac{\rho_{1}}{K_{o x}} *(L O H-H T)+\rho_{7} * Z}{\left(\rho_{2}+\rho_{1} *(L O O H+H P)\right.} \frac{{ }_{Z}^{C a}}{\Delta_{2}^{C a}} \\
& \rho_{1}=k_{o x}+k_{o x 2} \frac{C a}{K_{2}^{C a}} \\
& \rho_{2}=k_{r}+k_{r 2} \frac{C a}{K_{2}^{C a}} \\
& \rho_{7}=k_{\text {ing }}\left(1+\frac{C a}{K_{3}^{C a}}\right) \\
& \Delta_{Z}=1+\frac{Z}{K d z} \\
& { }_{z}^{C a}=1+\frac{Z}{K_{d z}}+\frac{C a}{K_{3}^{C a}}+\frac{Z}{K_{d z}} * \frac{C a}{K_{3}^{C a}} \\
& =\left(1+\frac{Z}{K_{d z}}\right) *\left(1+\frac{C a}{K_{3}^{C a}}\right) \\
& { }_{2}{ }_{2}^{C a}=1+\frac{C a}{K_{2}^{C a}}
\end{aligned}
$$

Since 5-LO undergoes irreversible inactivation, the concentration of active enzyme tends to zero with time and, consequently, all enzyme catalyzed reactions become equal to zero. In vivo, regulatory mechanisms control intracellular 5-LO production de novo [13]. To obtain non-zero steady state concentrations of 5-LO states and, consequently, to derive the rate equations describing the activities of 5-LO, we did not consider self-inactivation of the enzyme and have not taken into account processes responsible for 5-LO production de novo. Under these assumptions the total concentration of active enzyme $\left(F_{a}\right)$ is equal to total enzyme concentration and $F_{a}$ is a parameter of the model. Additionally, identification of model parameters using in vitro experimental data was performed on the basis of a model which takes into account self-inactivation of 5-LO with time (see Additional file 1: Appendix 1.5). Under these conditions $F_{a}$ represents the sum of active states of 5 -LO and changes with time. All rate equations were derived on the basis of the quasi-steady state approach [27].

\section{The kinetic model of phospholipase $\mathrm{A}_{2}$}

Calcium-dependent phospholipase A2 (cPLA2) catalyzes the production of AA from phospholipids (PL) in the cell membrane. Elevations in the cellular calcium concentration significantly stimulate cPLA2 activity. On the basis of several models of the enzyme developed previously and available experimental data $[28,29]$ we have derived the rate equation for CPLA2 to be $\left(V_{A A}^{P L A 2}\right.$, see Additional file 1: Appendix 2):

$$
V_{A A}^{P L A 2}=\frac{V_{\max }^{P L A 2} \cdot P L A 2_{2}^{C a} P L}{K_{m}^{P L A 2_{A P C}}+P L},
$$

where

$$
P L A 2_{2}^{C a}=\frac{C a}{K_{C a}^{P L A 2}+C a}
$$

\section{The kinetic model of glutathione peroxidase}

GPx enzyme reduces HP to HT. This reaction requires glutathione as a cofactor $[7,8]$. The stoichiometry of the reaction catalyzed by the enzyme is as follows:

$$
H P+2 G S H=H T=G S S G
$$

GPx catalyzes this reaction in accordance to the PingPong mechanism and the derivation of the rate equation for GPx is given in Additional file 1: Appendix 3:

$$
V^{G P x}=\frac{G P x_{f u l l} \cdot B}{A}
$$

where

$$
\begin{aligned}
A= & \frac{H P}{K_{m}^{H P}} \cdot\left(1+\frac{H T}{K_{H T}^{G P x}}\right)+\frac{G S H}{K_{m}^{G S H}} \cdot \frac{G S H}{K_{m}^{G S H}} \cdot\left(1+\frac{G S S G}{K_{G S S G}^{g p x}}\right) \\
& +\frac{G S H}{K_{m}^{G S H}} \cdot \frac{G S H}{K_{m}^{G S H}} \cdot \frac{H P}{K_{m}^{H P}} \\
B= & \frac{k_{c a t}^{G P x} \cdot H P}{K_{m}^{H P}} \frac{G S H}{K_{m}^{G S H}} \frac{G S H}{K_{m}^{G S H}}
\end{aligned}
$$

The kinetic model of 5-hydroxyeicosanoid dehydrogenase HEDH catalyzes the conversion of HT into oxoETE. The mechanism of $\mathrm{HEDH}$ is considered as Bi-Bi Ping-Pong, with NADP as the second substrate [9]. Derivation of 
the rate equation for HEDH is given in Additional file 1: Appendix 4:

$$
V^{H E D H}=\frac{H E D H_{\text {full }} B}{A}
$$

where

$$
\begin{aligned}
& B=\frac{k_{1}^{\text {hedh }} k_{2}^{\text {hedh }} H T \text { NADP }}{K_{H T}^{\text {hedh }} K_{N A D P}^{\text {hedh }}} \\
& -\frac{k_{-1}^{\text {hedh }} k_{-2}^{\text {hedh }} \text { oxoETE NADPH }}{K_{\text {oxoETE }}^{\text {hedh }} K_{N A D P H}^{\text {hedh }}} \\
& A=\left(1+\frac{N A D P}{K_{N A D P}^{\text {hedh }}}+\frac{\text { oxoETE }}{K_{\text {oxoETE }}^{\text {hedh }}}\right) \\
& \cdot\left(\frac{k_{1}^{\text {hedh }} H T}{K_{H T}^{\text {hedh }}}+\frac{k_{-2}^{\text {hedh }} N A D P H}{K_{N A D P H}^{\text {hedh }}}\right) \\
& +\left(1+\frac{H T}{K_{H T}^{h e d h}}+\frac{N A D P H}{K_{N A D P H}^{h e d h}}\right) \\
& \cdot\left(\frac{k_{-1}^{\text {hedh }} \text { oxoETE }}{K_{\text {oxoETE }}^{\text {hedh }}}+\frac{k_{2}^{\text {hedh }} N A D P}{K_{N A D P}^{\text {hedh }}}\right)
\end{aligned}
$$

The LTA4 and oxoETE synthesis model ("LOS model")

To build the "LOS model" we utilized the rate equations describing the activities of 5-LO, phospholipase A2, glutathione peroxidase and 5-hydroxyeicosanoid dehydrogenase as given above. The kinetic scheme of the "LOS model" is shown in Figure 1. In accordance with the scheme, AA binding to 5-LO is converted to HP via the lipoxygenase reaction. As a result a complex of 5-LO and HP is formed. HP can be either released from the complex $\left(V_{H P s y n}^{5 L O}\right)$ or used to form LTA4 via the LTA4synthase reaction $V_{L T A s y n}^{L L O}$. Additionally, LTA4 can be produced from free HP in the absence of AA (see sequence of reversible reaction $V_{H P s y n}^{5 L O}$ and irreversible reaction $\left.V_{L T A s y n}^{5 L O}\right)$. To present all these process correctly the intermediate state of the enzyme ${ }^{(C a)} F_{H P}^{(A A)}$ (complex 5-LO with HP) was added to the kinetic scheme. The concentration of the state ${ }^{(C a)} F_{H P}^{(A A)}$ is not a variable of the model, i.e. there are no differential equations describing the time dynamics of ${ }^{(C a)} F_{H P}^{(A A)}$. However, in accordance with the quasi-steady state approach chosen to describe 5-LO operation in the "LOS model" (and, consequently, applied to derive rate equations of various 5-LO activities) concentration of state ${ }^{(C a)} F_{H P}^{(A A)}$ is expressed in terms of variables of the "LOS model" (see Additional file 1: Appendix 1).

To avoid unlimited accumulation of metabolites resulting from constant influx of AA we have introduced processes of degradation of HT, oxoETE and LTA4 $\left(V_{H T d}, V_{\text {LTAd }}\right.$ and $\left.V_{\text {oxoETEd }}\right)$ in the model. The reaction rates of these processes are described in accordance with mass action law (Additional file 1: Appendix 5). Additionally, concentrations of $\mathrm{PL}$, lipid peroxide $\mathrm{LOOH}$ and its reduced product $\mathrm{LOH}$, reduced (GSH) and oxidized (GSSG) glutathione, and reduced and oxidized forms of NADPH are considered as parameters of the model, i.e., do not change with time. The values for the intracellular concentrations of GSH, GSSG, $\mathrm{NADPH}$ and NADP were taken from the following sources [30-34]. The concentration of $\mathrm{LOOH}$ has either been chosen on the basis of known experimental conditions or has been varied to describe various oxidative states of the cells.

Based on all the above assumptions the system of differential equations describing the "LOS model" is presented below:

$$
\begin{aligned}
& \frac{d A A}{d t}=V_{A A}^{P L A 2}-V_{A A c o n s}^{5 L O} \\
& \frac{d H P}{d t}=V_{H P s y n}^{5 L O}-V_{H P c o n s}^{5 L O}-V^{G P x} \\
& \frac{d H T}{d t}=V_{H P c o n s}^{5 L O}+V^{G P x}-V^{H E D H}-V_{H T d} \\
& \frac{d L T A 4}{d t}=V_{L T A s y n}^{5 L O}-V_{L T A d} \\
& \frac{d o x o E T E}{d t}=V^{H E D H}-V_{\text {oxoETEd }}
\end{aligned}
$$

\section{Description of the parameters of the "LOS model" and experimental data used for their identification}

According to assumption L of the section "Known experimental data and hypotheses used for the model development", some parameters were equated with each other (see 1.4). Thus, for 5-LO 17 independent parameters remained, among them 11 equilibrium constants and 6 rate constants. Additionally, 5 parameters for GPx, and 8 parameters for $\mathrm{HEDH}$ needed to be identified. Several of the values of the parameters have been directly taken from other literature sourcese.g. the Michaelis constant for glutathione $\left(K_{m}^{G S H}\right)$ for glutathione peroxidase reaction [7], the rate constant of LTA4 and HT degradation [23]. The values for other parameters were chosen on the basis of the best coincidence between modeling results and corresponding experimental data. To select the values of the parameters we used the algorithm of fitting based on the Hook-Jeeves method [35] implemented in the DBSolve Optimum package [36]. As a criterion of fitness, the following function was used:

$$
f\left(k_{j}, K_{j}\right)=\sum_{i}^{n}\left(v_{i}-\bar{v}_{i}\right)^{2}
$$

Here, $n$ is the total number of experimental points, ${ }_{i} \bar{v}$ is the experimentally measured value of the variable or reaction rate, $v_{i}$ is the value of the variable or reaction 
rate calculated based on the model at a point corresponding to the experimental ones.

Given the complexity of the model, simultaneous identification of parameter estimates would be challenging. Therefore, parameter identification was performed individually for each enzyme by fitting to literature data sets pertinent to the specific enzyme. For example, the parameters of 5-LO were identified via fitting of the 5-LO model against more than 10 experimentally measured curves (76 experimental points) [22,24-26,37,38], 4 unknown parameters of GPx were identified on the basis of 12 experimentally measured points [39] and the parameters of HEDH have been fitted against 47 experimental points [9].

\section{Results and discussion Modeling of 5-LO kinetics}

The "LOS model" and parameter values identified during model building enabled us to reproduce various experimental data on the kinetics of 5-LO. Due to the large number of fits only selected representative examples are presented in the main text. The values of the parameters obtained are given in Table 1. Figure 4 demonstrates a model generated curve fitted to the experimentally measured dependencies of HP production rate on AA [24]. Based on this it was concluded that the model of 5-LO satisfactorily described the observed non-monotonic behavior. Figure 5 demonstrates the simulated time series of total concentration of HP and HT fitted to literature data [37]. Additionally, our model satisfactorily fitted experimental data on LTA4 production from endogenous and exogenous HP (see Figure 6) [26] and the $\mathrm{Ca}^{2+}$ dependence of 5-HP production by $5-\mathrm{LO}$ on $\mathrm{Ca}^{2+}$ concentration [38]. In the latter example the model satisfactorily reproduced both the EC50 value $(2-3 \mu \mathrm{M})$ and the non-monotonic shape of the experimentally measured dependence (see Figure 7). Other results of fitting are summarized in Additional file 1: Appendix 6.

The next step of model evaluation examined its ability to reproduce experimentally measured data which had not been used for parameter identification. As an example we selected a dataset describing the influence of glutathione on 5-LO. In accordance with the experimental data [40], the effect of glutathione on 5-LO products in a cell-free extract strongly depends on the concentration of AA. Under conditions of low AA concentrations, the reaction rate of 5-LO is inversely proportional to the glutathione concentration. When AA concentration is high, the rate of 5-LO catalyzed reactions do not decrease even at high concentrations of GSH. Figure 8 demonstrates that our "LOS model" satisfactorily reproduces this threshold influence of GSH on the total concentration of "5-LO metabolites" (sum of HP, HT and LTA4) at various concentrations of AA. Our model
Table 1 Kinetic parameters of enzyme catalytic cycles

\begin{tabular}{|c|c|c|}
\hline Parameter & Value & Units \\
\hline$V_{\max }^{P L A 2}$ & 450 & $\mu \mathrm{M} / \mathrm{min}$ \\
\hline$K_{m}^{P L A 2}{ }^{P A P C}$ & 20 & $\mu \mathrm{M}$ \\
\hline$K_{C a}^{P L A 2}$ & 0.1 & $\mu \mathrm{M}$ \\
\hline$K_{A A}$ & 10.7 & $\mu \mathrm{M}$ \\
\hline$K_{2}^{C a}$ & 7.11 & $\mathrm{mM}$ \\
\hline$K_{2}^{C a}$ & 14.4 & $\mu \mathrm{M}$ \\
\hline$K_{o x}$ & 100 & $\mu \mathrm{M}$ \\
\hline$K_{r}$ & 5.8 10-7 & - \\
\hline$K_{A A}^{i}$ & 552 & $\mu \mathrm{M}$ \\
\hline$K_{H T}$ & 0.54 & $\mu \mathrm{M}$ \\
\hline$K_{d 3}$ & $1.310-4$ & - \\
\hline$k_{10}$ & 4.6103 & $1 / \min$ \\
\hline$k_{3}$ & 0.34103 & $(\min \cdot \mu M)^{-1}$ \\
\hline$k_{o x}$ & 2.7 10-4 & $(\min \cdot \mu M)^{-1}$ \\
\hline$k_{0 \times 2}$ & 67.2 & $(\min \cdot \mu M)^{-1}$ \\
\hline$k_{r}$ & 2.54 10-4 & $1 / \min$ \\
\hline$k_{r 2}$ & $4.410-5$ & $1 / \min$ \\
\hline$k_{\text {LTAsyn }}$ & 5.4 10-4 & $1 / \min$ \\
\hline$k_{\text {ing }}$ & 16450 & $1 / \min$ \\
\hline$K_{d z}$ & 36.6 & $\mu \mathrm{M}$ \\
\hline$K_{P F}$ & 0.126 & $\mathrm{uM}$ \\
\hline$\overline{K G S S G}$ & 0.072 & $\mu \mathrm{M}$ \\
\hline$k_{c a t}^{g p x}$ & 0.489 & $1 / \min$ \\
\hline$K_{N A D P}^{\text {hedh }}$ & 2.9 & $\mu \mathrm{M}$ \\
\hline$K_{N A D P H}^{h e d h}$ & 2.69 & $\mu \mathrm{M}$ \\
\hline KoxoETE & 1.67 & $\mu \mathrm{M}$ \\
\hline$\overline{K_{H T}^{h e d h}}$ & 0.332 & $\mu \mathrm{M}$ \\
\hline$k_{1}^{\text {hedh }}$ & 88.34 & $1 / \min$ \\
\hline$k_{2}^{\text {hedh }}$ & 1724 & $1 / \min$ \\
\hline$k_{-1}^{\text {hedh }}$ & 31.5 & $1 / \min$ \\
\hline $\bar{k}$ & 8.08 & $1 / \mathrm{min}$ \\
\hline$k_{H T d}$ & 0.001 & $1 / \min$ \\
\hline$k_{\text {LTAd }}$ & 0.07 & $1 / \min$ \\
\hline$k_{\text {OXOETEd }}$ & 0.005 & $1 / \min$ \\
\hline
\end{tabular}

explains this phenomenon (threshold like response to GSH increase at various AA concentrations) as driven by the lack of lipid peroxides in this cell-free extract system under conditions of low AA concentrations. In this case the main agent responsible for 5 -LO oxidation is HP (see reaction $\mathrm{r} 6$ in Figure 3). HP acts as an electron acceptor in the pseudo-peroxidase reaction converting 5-LO from inactive $\left(\mathrm{Fe}^{2+}\right)$ to active $\left(\mathrm{Fe}^{3+}\right)$ state. Under conditions of low AA concentrations, 5-LO is unable to produce a sufficient quantity of HP molecules to drive the transition of the enzyme to active form. Additionally data from experiments with PMN homogenates [40] demonstrated that the addition of GSH can, however, suppress the 


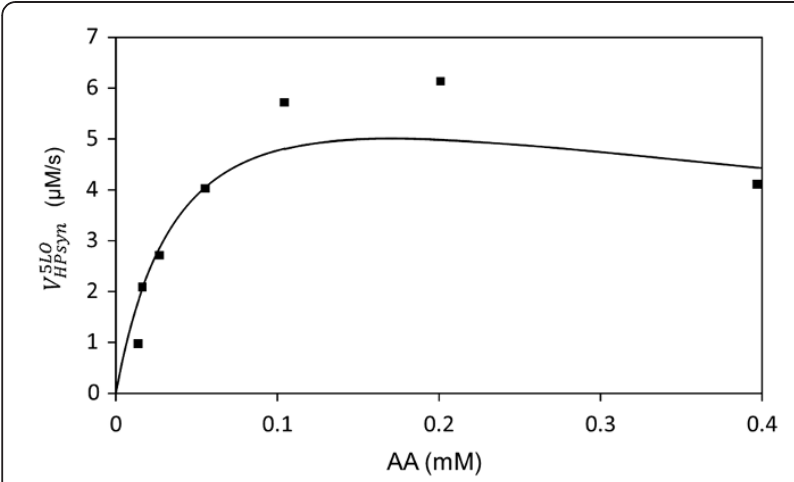

Figure 4 Dependence of rate of HP synthesis on AA concentration [24]. Experimental conditions: $0.2 \mathrm{mM}$ ATP, $0.3 \mathrm{mM}$ $\mathrm{CaCl}$. Dots correspond to experimental data; solid line is model generated curve.

activation of 5-LO, as long as the AA concentration remains below a critical limit.

\section{Modeling of the response to redox and non-redox inhibitors of 5-LO}

We applied the "LOS model" to study the influences of redox and non-redox inhibitors on the operation of 5LO. Inhibitor PF has been profiled in stimulated human whole blood (HWB) against several relevant human targets including 5-LO, 12-LOX, 15-LO and COX enzymes [18]. The compound completely inhibited the synthesis of the 5-LO products (HT, oxoETE, LTB4 and LTE4) with estimated IC50s between 100 and $190 \mathrm{nM}$. These data demonstrate that the non-redox inhibitor PF is 6-10 times more potent than zileuton.

The mechanism of 5-LO inhibition by the redox and non-redox inhibitors has been modeled under LTA4 steady-state concentration (Additional file 1: Appendix 1).

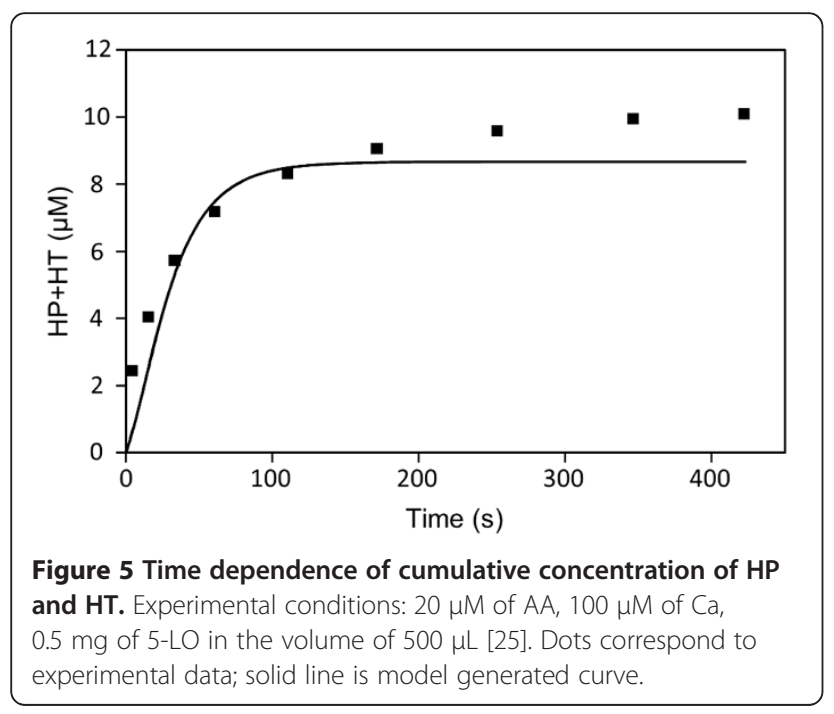

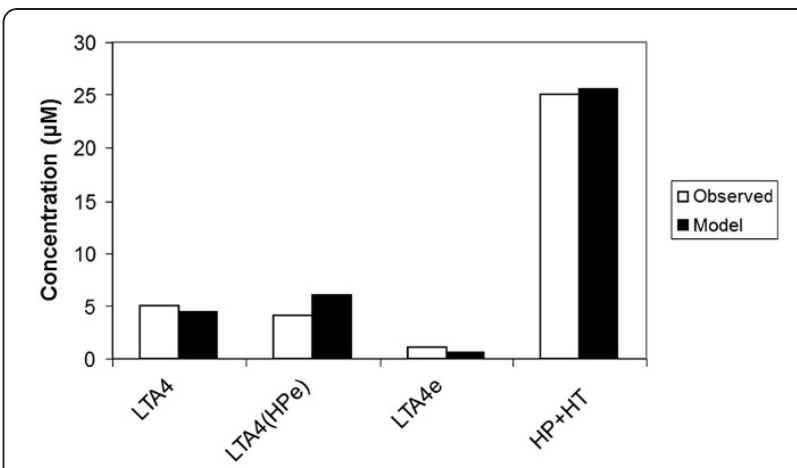

Figure 6 The effect of exogenous HP on the formation of deuterated LTA4 hydrolysis products. Human leukocyte homogenate supernatant was incubated with $100 \mu \mathrm{M}$ octadeuterated arachidonic acid and $80 \mu \mathrm{M}$ of the exogenous HP, from left to right: LTA4 in the absence of endogenous HP, deuterated LTA4 in presence of exogenous HP, LTA4 formed from exogenous HP, total amount of 5-LO products HP and HT in the absence of exogenous HP.

Parameter values describing the kinetic properties of redox and non-redox 5-LO inhibitors were chosen in such a way to provide satisfactory coincidence between the IC50 and IC80 measured experimentally [18] and those calculated by the "LOS model".

To validate the model describing 5-LO we again utilized comparison of test data sets to simulated outcomes. Examples of such "independent" data sets were

(i) time dependences of HP and HT measured at various zileuton concentrations in in vitro experiments with 5-LO [3].

(ii) time dependences of peroxides measured in the presence and absence of $\mathrm{PF}$ and zileuton in ex vivo experiments [18].

Figure 9 demonstrates that the derived values of parameters (Table 1) for zileuton on 5-LO allowed the model to satisfactorily reproduce experimentally measured production of the sum of HP and HT [3]. Moreover, the "LOS model" qualitatively reproduced experimental kinetic data describing the application of redox and non-redox inhibitors in vitro. Experiments in a crude cell lysates 5 -LO system containing $10 \mu \mathrm{M}$ of peroxide (13(S)-HpODE) examined the addition of $10 \mu \mathrm{M}$ of zileuton or PF (reference). The "LOS model" satisfactory reproduced peroxide consumption following zileuton, and the absence of effect following PF application (Figure 10) due to its non-redox mechanism [18].

Thus, comparison of the model simulations to two sets of experimental data which were not used in parameter identification enabled us to conclude that our model adequately described the influence of inhibitors on the system behavior. 


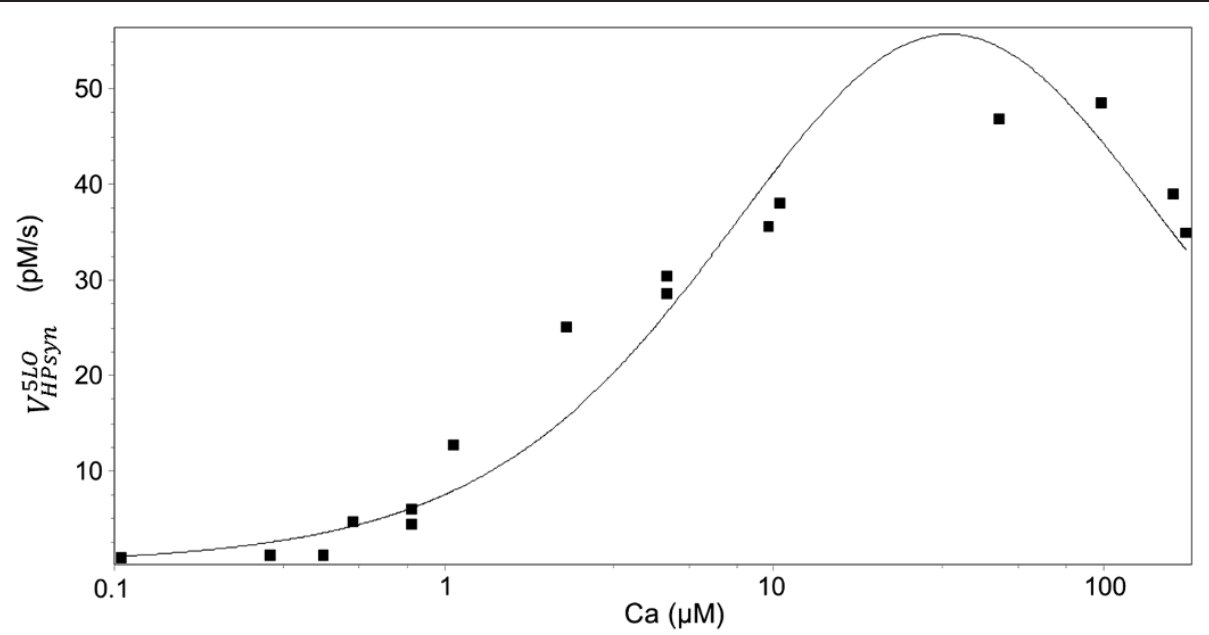

Figure 7 Dependence of the HP production on the Ca concentration. Experimental conditions: $20 \mu \mathrm{M}$ of AA, 0.1 mM ATP. Dots correspond to experimental data from [38], line correspond to results of calculations.

\section{Difference between redox and non-redox inhibitors}

As have already been discussed earlier, the redox state of the cell (GSH and lipid peroxide concentration) is able to significantly influence 5-LO activity. In the "LOS model" different levels of oxidative stress were described in terms of $\mathrm{LOOH}$ (representing total peroxide concentration except HP). To predict system responses to redox and non-redox inhibitors and to identify possible differences between them at different levels of $\mathrm{LOOH}$ we simulated the dependence of steady state LTA4 and oxoETE on PF and zileuton concentration. Several $\mathrm{LOOH}$ concentrations were chosen for this study: $0 \mu \mathrm{M}$ (no oxidative stress), $5 \mu \mathrm{M}$ (normal level according to available literature data [41]), $10 \mu \mathrm{M}$ (asthmatic patients

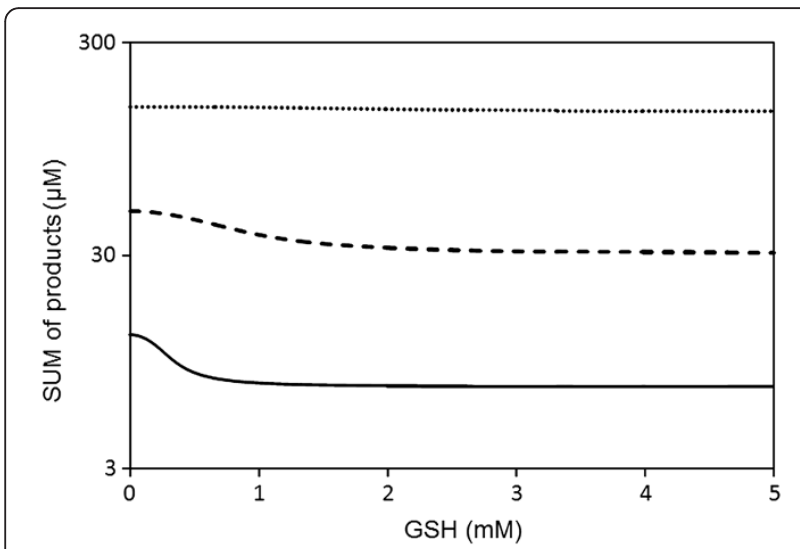

Figure 8 Simulation of influence of glutathione on production of 5-LO metabolites (sum of LTA4, HP and HT). Parameters of the LOS model used for simulation: pool of NADP 3 mM; NADPH 2 mM; Ca $1 \mathrm{mM}$; 5-LO $0.1 \mu \mathrm{M}$; HEDH5 $0.1 \mu \mathrm{M}$; Values of parameter PL responsible for steady state AA level are 117 (solid), 120 (dash), 125 (dot).
[41]) and $100 \mu \mathrm{M}$ (extremely high oxidative stress). For all simulations the system was run to steady-state conditions with subsequent addition of inhibitor and the effect at the new steady-state noted. This was represented as the percentage of inhibition of LTA4 and oxoETE at a given inhibitor concentration and was calculated using the following equations:

$$
\begin{gathered}
I n h_{\%}^{L T A 4}=100\left(1-\frac{\left[L T A 4_{s t}^{i n h}\right]}{\left[L T A 4_{s t}^{i n h}=0\right]}\right) \\
I n h_{\%}^{o x o E T E}=100\left(1-\frac{[\text { oxoETE }}{\left[o x o E T E_{s t}^{i n h}\right]}\right]
\end{gathered}
$$

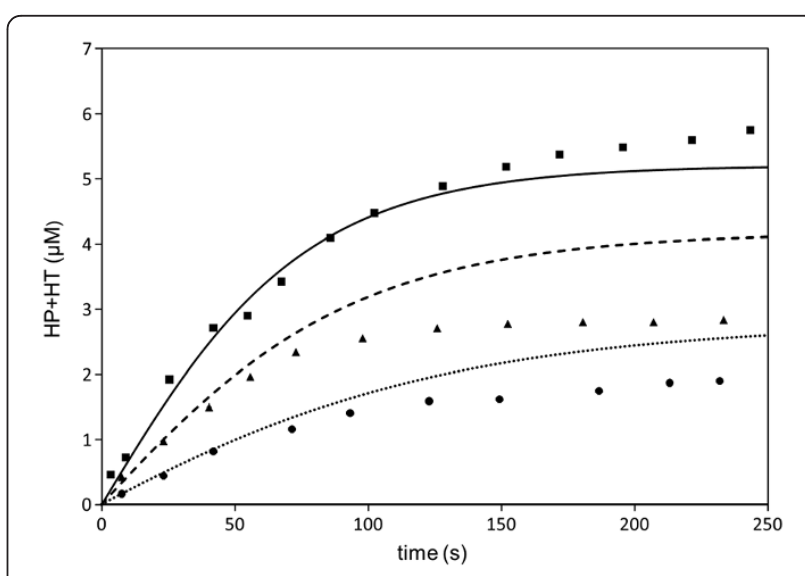

Figure 9 Dependence of sum of the concentrations of HP and HT on time in presence of zileuton. Experimental conditions: with no 5-LO inhibitor applied ((squares - data, solid line - model

results), with $5 \mu \mathrm{M}$ of Zileuton applied (triangles - data, dashed line - model results)) and with $20 \mu \mathrm{M}$ of Zileuton applied (circles - data, dotted line - model results) [3]. Other concentrations: $20 \mu \mathrm{M}$ of AA, $0.4 \mathrm{mM}$ of $\mathrm{Ca}$. 


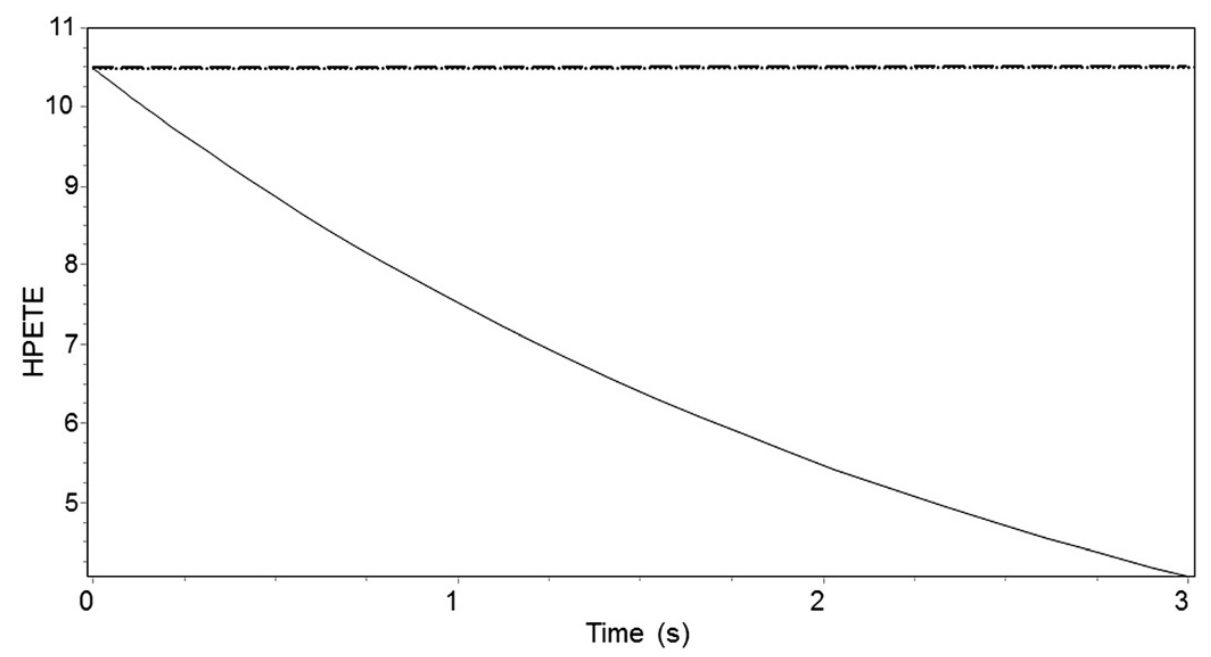

Figure 10 Quantitative simulation of experiment [18] with different inhibitors. Consumption of $10 \mu \mathrm{M} \mathrm{HP}$ by 5-LO system in presence of $10 \mu \mathrm{M}$ PF (dotted line) or $10 \mu \mathrm{M}$ Zileuton (solid line) or in the absence of inhibitors (dashed line, coinciding with dotted line). Concentrations: PL $40 \mu \mathrm{M}$, pool of glutathione $10 \mathrm{mM}, \mathrm{GSH} 5 \mathrm{mM}$. Other parameters are as in the legend to Figure 8.

where $L T A 4_{s t}^{i n h}$, oxoETE $E_{s t}^{i n h}$, are steady-state concentrations of LTA4 and oxoETE at a specified inhibitor concentration $L T A 4_{s t}^{i n h}=0$, oxoETE $E_{s t}^{i n h}=0$ are steady-state concentrations of LTA4 and oxoETE in the absence of inhibitor.

We have simulated how the LTA4 and oxoETE dose response depends on the $\mathrm{LOOH}$ level and found that both were influenced significantly (Table 2). Indeed, assuming $\mathrm{LOOH}$ level equal to 0 (no oxidative stress) we have simulated how steady state concentrations of LTA4 and oxoETE depend on zileuton and PF compound concentrations (Figures 11-12, solid lines). Figure 11 demonstrates that model fits satisfactorily ex vivo experimental data [18] on the dependence of LTA4 and oxoETE on PF concentration (Tables 3,2 ). The solid lines on Figures 11-12 also show that the potency of zileuton to inhibit oxoETE production is the same as for LTA4 production, but it is ten times lower than the potency of PF compound to inhibit both rates (see Tables 3, 2).

Assuming a level of $\mathrm{LOOH}$ in healthy controls of $5 \mu \mathrm{M}$ (see Figures 11-12, (dashed lines)) we have found

Table 2 Potency of 5-LO Inhibitors in "LOS" model at different peroxide concentrations

\begin{tabular}{lccccc}
\hline $\begin{array}{l}\text { Peroxide } \\
\begin{array}{l}\text { concentration } \\
(\mu \mathrm{M})\end{array}\end{array}$ & \multicolumn{2}{l}{$\begin{array}{l}\text { IC50 for inhibition by } \\
\text { Zileuton }(\mathrm{nM})\end{array}$} & & \multicolumn{2}{l}{$\begin{array}{l}\text { IC50 for inhibition by } \\
\text { PF-4191834(nM) }\end{array}$} \\
\cline { 2 - 3 } \cline { 5 - 6 } \cline { 5 - 6 } & For LTA4 & For 5oxoETE & & For LTA4 & For 5oxoETE \\
\hline $5^{*}$ & 980 & 962 & & 122 & 125 \\
\hline $10^{* *}$ & 860 & 1145 & & 125 & 129 \\
\hline 100 & 1450 & 1410 & & 143 & 146 \\
\hline
\end{tabular}

*- corresponds to healthy subjects.

** - corresponds to asthmatic subjects. that curves describing level of inhibition of LTA4 and oxoETE show a similar tendency but in case of inhibition with zileuton a new feature was observed. The increase in $\mathrm{LOOH}$ level from 0 to $5 \mu \mathrm{M}$ was accompanied by a decrease in zileuton potency for inhibition of oxoETE synthesis (see Figure 12, dashed line) in comparison with its

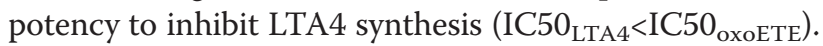
This effect is more pronounced when setting the [LOOH] equal to $10 \mu \mathrm{M}$ (approximating that of asthmatic patients, Figures 11-12, dotted lines). To further illustrate this effect we simulated the impact of a supra-physiological LOOH concentration of $100 \mu \mathrm{M}$ on the inhibitory properties of compounds (dash-dot lines in Figures 11b and 12b).

\section{Discussion of redox-inhibitor properties at high oxidative stress}

Zileuton donates an electron to the active $\left(\mathrm{Fe}^{3+}\right)$ state of 5-LO to form the inactive ferrous $\left(\mathrm{Fe}^{2+}\right)$ state and a zileuton radical (see reaction $r_{7}$ in Figure 3 ). The zileuton radical is eliminated without subsequent reduction to zileuton [42]. To activate 5-LO it is necessary to oxidize the inactive ferrous $(2+)$ state, i.e. an electron donated to an acceptor such as HP or LOOH. Therefore, under conditions of low $\mathrm{LOOH}$ only $\mathrm{HP}$ is able to oxidize 5-LO. If zileuton is applied, 5-LO is converted to the inactive ferrous state, the rate of $\mathrm{HP}$ synthesis decreases and, as a consequence, the enzyme is completely inhibited because of lack of its oxidation. In the absence of other sources of lipid peroxides and HP, 5-LO is completely inhibited by zileuton.

Under conditions of high $\mathrm{LOOH}$, the transformation of ferrous 5-LO state into ferric state can proceed in two 

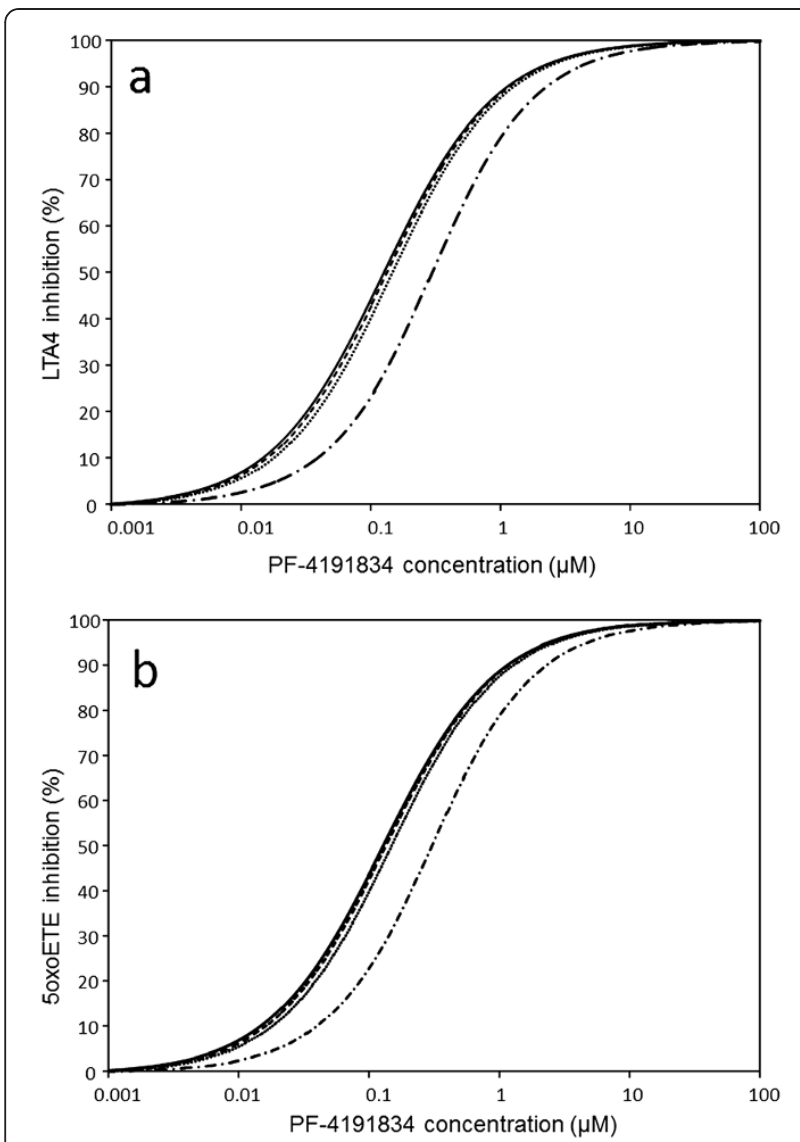

Figure 11 Simulation of influence of PF on LTA4 and oxoETE production. Concentrations of lipid peroxide ( $\mathrm{LOOH}$ ): 0 (solid), $5 \mu \mathrm{M}$ (dash), $10 \mu \mathrm{M}$ (dot), $100 \mu \mathrm{M}$ (dash-dot). Other parameters are as in the legend to Figure 10.

possible ways: either via HP or LOOH reduction (see reaction $r_{6}$ in Figure 3). When zileuton is applied, the concentration of ferrous state of 5-LO increases. However, high level of LOOH may compensate for the lack of HP in the pseudo-peroxidase reaction that leads to maintenance of level of the active 5-LO state and, as a consequence, the lipoxygenase reaction occurs. Under these conditions the rate of pseudo-peroxidase reaction increases and both $\mathrm{HP}$ and $\mathrm{LOOH}$ are reduced in this reaction converting 5-LO into active ferric state. As a
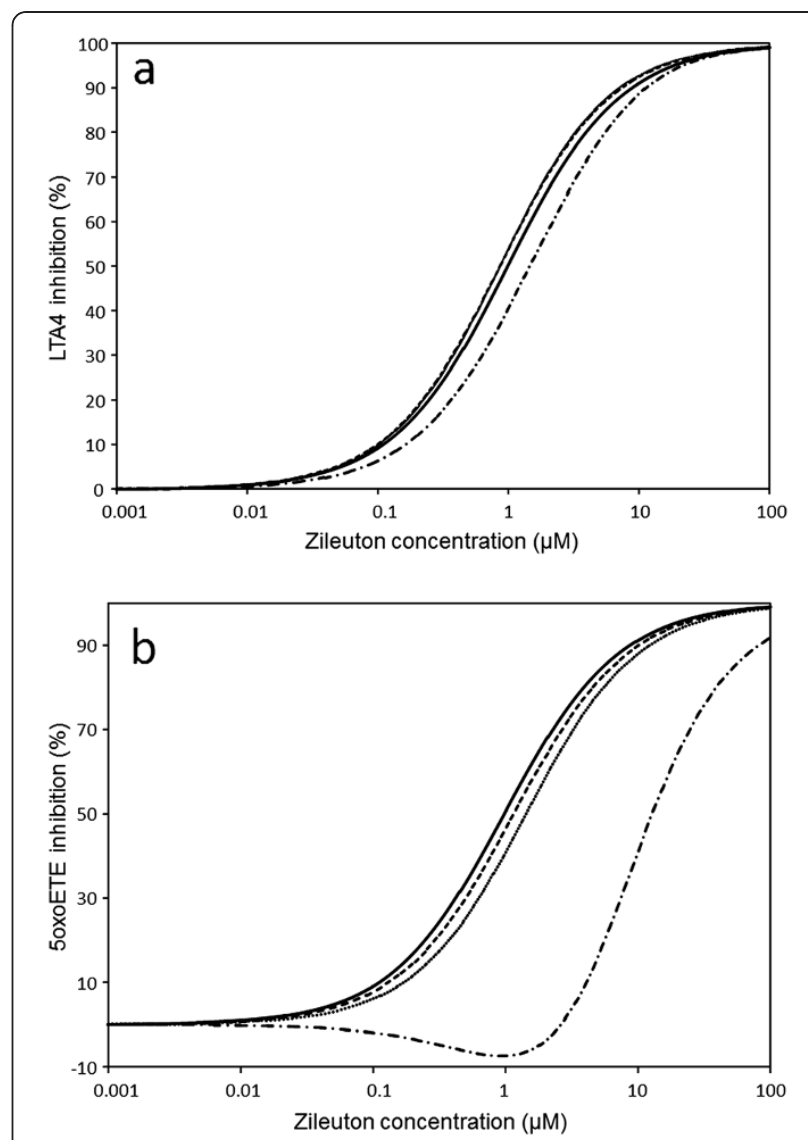

Figure 12 Simulation of influence of zileuton on LTA4 and oxoETE production. Concentrations of lipid peroxide ( $\mathrm{LOOH})$ : 0 (solid), $5 \mu \mathrm{M}$ (dash), $10 \mu \mathrm{M}$ (dot), $100 \mu \mathrm{M}$ (dash-dot). Other parameters are as in the legend to Figure 10. consequence of increased HP consumption in the pseudo-peroxidase reaction, its concentration decreases, leading to a decrease in LTA4 production. At the same time increases in HT concentration produced in the pseudo-peroxidase reaction leads to further increase in oxoETE production.

\section{Conclusions}

We have constructed a model of 5-LO activity and regulation that demonstrates emergent properties consistent

Table 3 Potency of 5-LO inhibitors in whole blood from control (Normal) and asthmatic human volunteer [18]

\begin{tabular}{|c|c|c|c|c|c|c|c|c|}
\hline & \multicolumn{4}{|c|}{ CONTROL } & \multicolumn{4}{|c|}{ ASTHMATICS } \\
\hline & \multicolumn{2}{|c|}{ IC50 (nM) } & \multicolumn{2}{|c|}{ IC80 (nM) } & \multicolumn{2}{|c|}{ IC50 (nM) } & \multicolumn{2}{|c|}{ IC80 (nM) } \\
\hline & Experim. & Model & Experim. & Model & Experim. & Model & Experim. & Model \\
\hline Zileuton & $850 \pm 220$ & 860 & $2270 \pm 710$ & 3330 & $1080 \pm 220$ & 860 & $2520 \pm 1010$ & 3270 \\
\hline PF-4191834 & $120 \pm 10$ & 125 & $300 \pm 20$ & 518 & $180 \pm 20$ & 143 & $390 \pm 50$ & 550 \\
\hline
\end{tabular}


with a wide range of experimental data. Utilizing this model we have derived several conclusions:

i) Both redox and non-redox inhibitors of 5-LO decrease LTA4 production independently of lipid peroxide level.

ii) Non-redox inhibitors of 5-LO decrease HT production (and, consequently, 5-oxoETE) independently of lipid peroxide level.

iii) The effect of redox inhibitors on oxoETE production strongly depends on $\mathrm{LOOH}$ level;

iv) Redox inhibition of 5- $\mathrm{LO}$ under condition of high LOOH switches 5-LO from LTA4 production to HT (and, consequently, oxoETE) production.

OxoETE is one of the key inflammatory mediators and the results of our work presented in the paper have demonstrated that under certain conditions redox inhibitors can lead to a non-intuitive increase in oxoETE synthesis. Together with the PK disadvantages of zileuton this observation supports the further investigation of non-redox inhibitors as possible drugs against asthma.

\section{Additional files:}

Additional file 1: Appendix 1. Derivation of model equations.

Additional file 2: Appendix 2. Additional figures.

\section{Abbreviations}

5-LO: 5-Lipoxygenase; AA: Arachidonic acid; HP: 5(S)-Hydroperoxy-6,8,11,14(E,Z,Z,Z)-eicosatetraenoic acid; LTA4: Leukotriene A4; HT: 5-

Hydroxyeicosatetraenoic acid; PL: Phospholipids; PLA2: Phospholipase A2; GPx: Glutathione peroxidase 1; GSH: Glutathione; HEDH: 5-Hydroxyeicosanoid dehydrogenase; OxoETE: 5-Oxo-6E,8Z,11Z,14Z-eicosatetraenoic acid; PF: 4-(3-(4-(1-Methyl-1H-pyrazol-5-yl)phenylthio)phenyl)-tetrahydro-2H-pyran-4carboxamide; Z: Zileuton or N-(I-benzo(b)thien-2-ylethyl)-N-hydroxyurea), Zyflo IR/CR ${ }^{\circledR}$; LOOH: all other lipid peroxides except HP, which can be consumed by 5-LO; LOH: products of pseudoperoxydase reactions with lipid peroxides except HP.

\section{Competing interests}

The authors declare that they have no competing interests.

\section{Authors' contributions}

TK developed 5LO kinetic model; ODJ developed models for GPX and HEDH; KZ combined the models and described action of inhibitors, simulated inhibition curves; DS described action of inhibitors, simulated inhibition curves; OD, DF and BA took part in study design and combining the models. All authors took part in the conception of the manuscript, drafted and revised the manuscript.

\section{Acknowledgements}

We would like to thank Jaime L. Masferrer for discussion of our results and Nail Gizzatkulov for help with calculations.

\section{Author details}

${ }^{1}$ Institute for Systems biology SPb, Moscow, Russia. ${ }^{2}$ Clinical Pharmacology, Pfizer PGRD, Sandwich, UK. ${ }^{3}$ Now Clinical Pharmacology and DMPK, Medlmmune, Cambridge, UK. ${ }^{4}$ Pharmacokinetics, Dynamics and Metabolism, Pfizer PGRD, Sandwich, UK.

Received: 20 August 2012 Accepted: 18 October 2012 Published: 12 November 2012

\section{References}

1. Hammarstrom S: Leukotrienes. Ann Rev Biochem 1983, 52:355-77.

2. Batt DG: 5-lipoxygenase inhibitors and their anti-inflammatory activities. Prog Med Chem 1992, 29:1-63.

3. Falgueyret J-P, Hutchinson JH, Riendau D: Criteria for the identification of non-redox inhibitors of 5-lipoxygenase. Biochem Pharmacol 1993. 45:978-981.

4. Ford-Hutchinson AW, Gresser M, Young RN: 5-lipoxygenase. Ann Rev Biochem 1994, 63:383-417.

5. Werz O: 5-Lipoxygenase: cellular biology and molecular pharmacology. Curr Drug Targets Inflamm Allergy 2002, 1:23-44.

6. Rådmark O: Arachidonate 5-lipoxygenase. Prostaglandins Other Lipid Mediat 2002, 68-69:211-234.

7. Martínez JI, García RD, Galarza AM: The kinetic mechanism of glutathione peroxidase from human platelets. Thromb Res 1982, 27(2):197-203.

8. Chiu DT, Stults FH, Tappel AL: Purification and properties of rat lung soluble glutathione peroxidase. Biochim Biophys Acta 1976, 445:558-66.

9. Erlemann KR, Cossette C, Grant GE, Lee GJ, Patel P, Rokach J, Powell WS: Regulation of 5-hydroxyeicosanoid dehydrogenase activity in monocytic cells. Biochem J 2007, 403:157-65.

10. Powell WS, Rokach J: Biochemistry, biology and chemistry of the 5-lipoxygenase product 5-oxo-ETE. Prog Lipid Res 2005, 44:154-83.

11. Powell WS, Chung D, Gravel S: 5-Oxo-6,8,11,14-eicosatetraenoic acid is a potent stimulator of human eosinophil migration. J Immunol 1995, 154:4123-32.

12. Radmark O, Werz O, Steinhilber D, Samuelsson B: 5-Lipoxygenase: regulation of expression and enzyme activity. Trends Biochem Sci 2007 32:332-341.

13. Radmark O, Samuelsson B: Regulation of 5-lipoxygenase enzyme activity. Biochem Biophys Res Commun 2005, 338:102-110.

14. Riendeau D, Falgueyret JP, Guay J, Ueda N, Yamamoto S: Pseudoperoxidase activity of 5-lipoxygenase stimulated by potent benzofuranol and $\mathrm{N}$-hydroxyurea inhibitors of the lipoxygenase reaction. Biochem J 1991, 274:287-92.

15. Awni WM, Locke C, Dube LM, Cavanaugh JH: Evaluation of the diurnal variation in the pharmacokinetics of zileuton in healthy volunteers. J Clin Pharmacol 1997, 37:388.

16. Awni WM, Braeckman RA, Granneman GR, Witt G, Dubé LM: Pharmacokinetics and pharmacodynamics of zileuton after oral administration of single and multiple dose regimens of zileuton $600 \mathrm{mg}$ in healthy volunteers. Clin Pharmacokinet 1995, 29:22-3.

17. Joshi EM, Heasley BH, Chordia MD, Macdonald TL: In vitro metabolism of 2-acetylbenzothiophene: relevance to zileuton hepatotoxicity. Chem Res Toxicol 2004, 17:137-43.

18. Masferrer JL, Zweifel BS, Hardy M, Anderson GD, Dufield D, Cortes-Burgos L, Pufahl RA, Graneto M: Pharmacology of 4-(3-(4-(1-methyl-1H-pyrazol-5-yl) phenylthio) phenyl)-tetrahydro-2H-pyran-4-carboxamide (PF-4191834), a Novel Selective, non-redox, 5-Lipoxygenase Inhibitor Effective in Inflammation and Pain. J Pharmacol Exp Ther 2010, 334:294-301.

19. Aharony D, Ross L, Stein RL: Kinetic mechanism of guinea Pig neutrophil 5-lipoxygenase. J Biol Chem 1986, 261:11512-11519.

20. Ludwig P, Holzhutter H-G, Colosimo A, Silvestrini MC, Schewe T, Rapoport SM: A kinetic model for lipoxygenases based on experimental data with the lipoxygenase of reticulocytes. Eur J Biochem 1987, 168:325-337.

21. Aharony D, Redkar-Brown DG, Hubbs SJ, Stein RL: Kinetic studies on the inactivation of 5-lipoxygenase by $5(\mathrm{~S})$-hydroperoxyeicosatetraenoic acid. Prostaglandins 1987, 33:85-100.

22. Lepley RA, Fitzpatrick FA: Irreversible inactivation of 5-lipoxygenase by leukotriene A4. Characterization of product inactivation with purified enzyme and intact leukocytes. J Biol Chem 1994, 269:2627-2631.

23. Yang K, Ma W, Liang H, Ouyang Q, Tang C, Lai L: Dynamic simulations on the arachidonic acid metabolic network. PLoS Comput Biol 2007, 3:e55.

24. Pande AH, Moe D, Nemec KN, Qin S, Tan S, Tatulian SA: Modulation of human 5-lipoxygenase activity by membrane lipids. Biochemistry 2004 43:14653-14666.

25. De Carolis E, Denis D, Riendeau D: Oxidative inactivation of human 5lipoxygenase in phosphatidylcholine vesicles. Eur J Biochem 1996, 235:416-423.

26. Puustinen T, Scheffer MM, Samuelsson B: Endogenously generated 5-hydroperoxyeicosatetraenoic acid is the preferred substrate for human leukocyte leukotriene A4 synthase activity. FEBS Lett 1987, 217:265-268. 
27. Mogilevskaya E, Bagrova N, Plyusnina T, Gizzatkulov N, Metelkin E, Goryacheva E, Smirnov S, Kosinsky Y, Dorodnov A, Peskov K, Karelina T, Goryanin I, Demin O: Kinetic modeling as a tool to integrate multilevel dynamic experimental data. Methods Mol Biol 2009, 563:197-218.

28. Kramer RM, Roberts EF, Manetta JV, Hyslop PA, Jakubowski JA: Thrombininduced phosphorylation and activation of $\mathrm{Ca}(2+)$-sensitive cytosolic phospholipase A2 in human platelets. J Biol Chem 1993, 268:26796-26804.

29. Lukas TJ: A signal transduction pathway model prototype I: From agonist to cellular endpoint. Biophys J 2004, 87:1406-1416.

30. Smith $\mathrm{CV}$, Jones DP, Guenthner TM, Lash LH, Lauterburg BH: Compartmentation of glutathione: implications for the study of toxicity and disease. Toxicol Appl Pharmacol 1996, 140:1-12.

31. Bruynzeel PL, Kok PT, Viëtor RJ, Verhagen J: On the optimal conditions of LTC4 formation by human eosinophils in vitro. Prostaglandins Leukot Med 1985, 20:11-22.

32. Linden $M$, Håkansson L, Ohlsson $K$, Sjödin $K$, Tegner $H$, Tunek A, Venge $P$ : Glutathione in bronchoalveolar lavage fluid from smokers is related to humoral markers of inflammatory cell activity. Inflammation 1989, 13:651658.

33. Graham FD, Erlemann KR, Gravel S, Rokach J, Powell WS: Oxidative stressinduced changes in pyridine nucleotides and chemoattractant 5-lipoxygenase products in aging neutrophils. Free Radic Biol Med 2009, 47:62-71.

34. Sander BJ, Oelshlegel FJ Jr, Brewer GJ: Quantitative analysis of pyridine nucleotides in red blood cells: a single-step extraction procedure. Anal Biochem 1976, 71:29-36.

35. Hook R, Jeeves TA: Direct search solution of numerical and statistical problems. J ACM 1961, 8:212-229.

36. Gizzatkulov NM, Goryanin II, Metelkin EA, Mogilevskaya EA, Peskov KV, Demin OV: DBSolve Optimum: a software package for kinetic modeling which allows dynamic visualization of simulation results. BMC Syst Biol 2010, 4:109.

37. Rakonjac M, Fischer L, Provost P, Werz O, Steinhilber D, Samuelsson B, Rådmark O: Coactosin-like protein supports 5-lipoxygenase enzyme activity and up-regulates leukotrienee A4 production. Proc Nat Acad Sci USA 2006, 103:13150-13155.

38. Percival $D$, Denis $D$, Riendeau $D$, Gresser MJ: Investigation of the mechanism of non-turnover-dependent inactivation of purified human 5-lipoxygenase. Eur J Biochem 1992, 210:109-117.

39. Jakobsson PJ, Mancini JA, Riendeau D, Ford-Hutchinson AW: Identification and characterization of a novel microsomal enzyme with glutathionedependent transferase and peroxidase activities. J Biol Chem 1997, 272 (36):22934-9.

40. Hatzelmann A, Schatz M, Ullrich V: Involvement of glutathione peroxidase activity in the stimulation of 5-lipoxygenase activity by glutathionedepleting agents in human polymorphonuclear leukocytes. Eur I Biochem 1989, 180:527-533.

41. Cakmak A, Zeyrek D, Atas A, Selek S, Erel O: Oxidative status and paraoxonase activity in children with asthma. Clin Invest Med 2009, 32:E327-E334.

42. Charnulitrat W, Mason RP, Riendeau D: Nitroxide metabolites from alkylhydroxylamines and $\mathrm{N}$-hydroxyurea derivatives resulting from reductive inhibition of soybean lipoxygenase. J Biol Chem 1992, 267:9574-9579.

\section{Submit your next manuscript to BioMed Central and take full advantage of:}

- Convenient online submission

- Thorough peer review

- No space constraints or color figure charges

- Immediate publication on acceptance

- Inclusion in PubMed, CAS, Scopus and Google Scholar

- Research which is freely available for redistribution 